\title{
O CUIDADO DE ENFERMAGEM NO ALOJAMENTO CONJUNTO: UMA REVISÃO INTEGRATIVA*
}

Fernanda Demutti Pimpão ${ }^{1}$, Nalú da Costa Kerber ${ }^{2}$, Fabiane Ferreira Francioni ${ }^{3}$, Rosiane Filipin Rangel ${ }^{4}$, Wilson Danilo Lunardi Filho ${ }^{5}$

RESUMO: Trata-se de uma revisão integrativa que teve como objetivo identificar, na literatura brasileira, como é realizado o cuidado de enfermagem no Alojamento Conjunto e estabelecer sua relação com a Política Nacional de Atenção Integral à Saúde da Mulher. A busca foi realizada na Literatura Latino-Americana e do Caribe em Ciências da Saúde, Base de Dados de Enfermagem, Literatura Internacional em Ciências da Saúde e Scientific Electronic Library Online, a partir dos descritores alojamento conjunto e cuidados de enfermagem, sendo selecionados 11 estudos; não houve recorte temporal para seleção dos textos. Predominou um cuidado permeado de preconceito, discriminação e desumanização no qual a mulher não é percebida como sujeito de direitos, contrariando a Política Nacional de Atenção Integral à Saúde da Mulher. Compreende-se que a humanização do cuidado de enfermagem à puérpera permanece como um desafio à Enfermagem e às políticas públicas de saúde.

PALAVRAS-CHAVE: Cuidados de enfermagem; Alojamento conjunto; Políticas públicas de saúde; Enfermagem.

\section{NURSING CARE ON THE HOSPITAL WARD: AN INTEGRATIVE REVIEW}

ABSTRACT: This integrative literature review aimed to identify how nursing care is undertaken on the hospital ward, and establish its relationship with the National Policy for Comprehensive Healthcare for Women. The search was carried out in the Latin-American and Caribbean System on Health Sciences Literature, the Nursing Database, International Literature in Health Sciences, and the Scientific Electronic Library Online, based on the descriptors hospital ward and nursing care, with 11 studies selected; there was no time cut-off in selecting the texts. There was a predominance of care permeated with prejudice, discrimination and de-humanization, in which the woman is not perceived as a subject with rights, counteracting the National Policy for Comprehensive Healthcare for Women. It is understood that the humanization of nursing care for women who have recently given birth remains a challenge for Nursing and for public health policies. KEYWORDS: Nursing care; Hospital ward; Public health policy; Nursing.

\section{EL CUIDADO DE ENFERMERÍA EN EL ALOJAMIENTO CONJUNTO: UNA REVISIÓN INTEGRATIVA}

RESUMEN: Esta es una revisión integrativa que tuvo como objetivo identificar, en la literatura brasileña, cómo se realiza el cuidado de enfermería en el Alojamiento Conjunto, así como establecer su relación con la Política Nacional de Atención Integral a la Salud de la Mujer. La búsqueda fue realizada en la Literatura Latinoamericana y de Caribe en Ciencias de la Salud, Base de Datos de Enfermería, Literatura Internacional en Ciencias de la Salud y Scientific Electronic Library Online, por los descriptores alojamiento conjunto y cuidados de enfermería, siendo elegidos 11 estudios; no se consideró el aspecto temporal para selección de los textos. Ha predominado un cuidado permeado de prejuicio, discriminación y desumanización en el cual la mujer no es percibida como sujeto de derechos, contrariando la Política Nacional de Atención Integral a la Salud de la Mujer. Se conluye que la humanización del cuidado de enfermería a la puérpera sigue siendo un desafío para la Enfermería y a las políticas públicas de salud.

PALABRAS CLAVES: Cuidados de enfermería; Alojamiento conjunto; Políticas públicas de salud; Enfermería.

*Artigo desenvolvido durante a disciplina Programas e Políticas de Saúde do Programa de Pós-Graduação em Enfermagem da Universidade Federal do Rio Grande -FURG, 2010.

${ }^{1}$ Enfermeira. "Mestre em Enfermagem. Professora do Centro Universitário CESMAC e da SEUNE. Membro do Grupo de Estudos e Pesquisas em Organização do Trabalho da Enfermagem e Saúde - GEPOTES e do Núcleo de Estudos e Pesquisas em Saúde - NEPES da Universidade Federal do Rio Grande - FURG.

${ }^{2}$ Enfermeira. Doutora em Enfermagem. Professora da Escola de Enfermagem e do Programa de Pós-Graduação em Enfermagem da FURG. Líder do Grupo de Pesquisa Viver Mulher.

${ }^{3}$ Enfermeira. Doutora em Enfermagem. Professora da Escola de Enfermageme do Programa de Pós-Graduação em Enfermagem da FURG. Membro do Núcleo de Assistência de Enfermagem e Saúde de pessoas com doenças crônicas da Universidade Federal de Santa Catarina-NUCRON e do GEPOTES ${ }^{4}$ Enfermeira. Mestre em Enfermagem. Professora do Centro Universitário Franciscano - UNIFRA. Membro do Grupo de Estudos e Pesquisa em Empreendedorismo Social na Enfermagem e Saúde - GEPESES UNIFRA e GEPOTES.

${ }^{5}$ Enfermeiro. Doutor em Enfermagem. Professor da Escola e do Programa de Pós-Graduação em Enfermagem da FURG. Membro do GEPOTES.

Autor correspondente:

Fernanda Demutti Pimpão

Universidade Federal do Rio Grande

Rua Zalony, 479 - 96200-070 - Rio Grande-RS-Brasil

E-mail: fhernandapimpao@yahoo.com.br
Recebido: 11/12/2011

Aprovado: 31/01/2012

Cogitare Enferm. 2012 Jul/Set; 17(3):562-7 


\section{INTRODUÇÃO}

O Programa de Assistência Integral à Saúde da Mulher, lançado em 1983 pelo Ministério da Saúde (MS), desencadeado pelo movimento feminista e fortemente influenciado pelo movimento de Reforma Sanitária, permitiu a ampliação da assistência à população feminina, até então, centrada apenas no ciclo gravídico-puerperal ${ }^{(1-2)}$. Além disso, o Programa assumiu uma nova ótica de atenção à saúde, pautada na integralidade, no sentido de individualizar a assistência e, dessa forma, torná-la mais resolutiva ${ }^{(3)}$.

Em 2004, o MS elaborou um mecanismo para operacionalização deste Programa, a Política Nacional de Atenção Integral à Saúde da Mulher (PNAISM) a qual, em síntese, enfatiza a garantia de direitos, bem como a ampliação do acesso aos serviços de saúde, nos diferentes níveis de complexidade, buscando a qualidade e a humanização da atenção integral à mulher no Sistema Único de Saúde. Como efeito, essas medidas visam a contribuir na redução da morbidade e mortalidade feminina no Brasil, especialmente, por causas evitáveis ${ }^{(4)}$. Contudo, apesar da melhoria da cobertura e da assistência, os elevados índices de mortalidade materna, além de indicarem baixas condições socioeconômicas, dentre outras questões, advertem quanto à precariedade da atenção à saúde nesse grupo populacional ${ }^{(2)}$.

Estima-se que, aproximadamente, 536 mil mulheres morreram em decorrência de complicações no ciclo gravídico-puerperal no ano de 2005, em todo o mundo. Destas, $85 \%$ viviam em países subdesenvolvidos ${ }^{(2)}$. No Brasil, em 2003, foram registrados 1.572 óbitos maternos $^{(5)}$. A mortalidade no ciclo gravídico-puerperal, inclusive em decorrência de aborto, apesar de não estar entre as 10 primeiras causas de mortalidade na população feminina brasileira, representa um relevante problema de saúde pública, face ao fato da gravidez ser um evento fisiológico da mulher e que, portanto, não deveria culminar em doença, tampouco ter como desfecho a morte ${ }^{(2)}$.

$\mathrm{Na}$ atenção obstétrica e neonatal, a PNAISM visa a qualificar e humanizar a assistência de saúde à mulher, incluindo a assistência em situação de abortamento(4). Nessa perspectiva, tendo como foco o cenário do Alojamento Conjunto (AC), a Enfermagem como profissão comprometida com a melhoria de saúde da população pode e deve contribuir para a aplicação dessa política pública ao agregar, na sua prática, o cuidado humanizado e qualificado à gestante e ao binômio mãe/filho.
Diante do exposto, torna-se relevante voltar o olhar para o cuidado de enfermagem no sistema de AC, visto que esse período de internação da gestante-puérpera e do seu recém-nascido pode ser crucial para prevenir possíveis complicações, bem como para tratá-las precocemente. Portanto, esse estudo teve como objetivo identificar na literatura brasileira como é realizado o cuidado de enfermagem no espaço do AC e estabelecer sua relação com a PNAISM.

\section{MÉTODO}

Trata-se de um estudo descritivo realizado por meio de revisão integrativa da literatura a qual tem por finalidade resumir o conhecimento de um determinado assunto, bem como produzir conhecimentos novos, os quais não foram abordados ou aprofundados em pesquisas anteriores ${ }^{(6)}$. Para tanto, seguiram-se as cinco fases propostas por $\operatorname{Cooper}^{(6)}$, a saber: 1) formulação do problema; 2) coleta de dados; 3) avaliação dos dados; 4) análise e interpretação; e 5) apresentação dos resultados.

A busca bibliográfica ocorreu nas bases de dados da Biblioteca Virtual em Saúde e da Bireme: Literatura Latino-Americana e do Caribe (LILACS), Base de Dados de Enfermagem (BDENF), Literatura Internacional em Ciências da Saúde (MEDLINE) e Scientific Electronic Library Online (SciELO), a partir dos descritores alojamento conjunto e cuidados de enfermagem. A busca e a coleta bibliográfica foram realizadas na segunda quinzena de setembro de $2010 \mathrm{e}$ atualizada na primeira quinzena de setembro de 2011. Não houve recorte temporal para seleção dos estudos.

Para seleção dos textos foram considerados os seguintes critérios de inclusão: ser pesquisa primária - incluindo artigos, dissertações e teses; publicação científica no idioma português; disponível online, na íntegra, nas bases de dados pesquisadas; abordar a temática do cuidado de enfermagem à mulher no AC no título e/ou no resumo. A opção por publicação científica no idioma português justifica-se visto que o objetivo deste estudo é identificar na literatura brasileira como vem sendo realizado o cuidado de enfermagem no espaço do $\mathrm{AC}$ e estabelecer uma relação com a PNAISM.

Foram localizados, no total, 43 estudos, assim distribuídos: 23 na LILACS, 15 na BDENF, 2 na MEDLINE e 3 na SciELO. Na LILACS somente 11 textos estavam disponíveis na íntegra, dos quais foram excluídos dois por não se adequaram ao objeto em estudo - um tratava do uso de fototerapia no neonato e outro sobre 
a construção de um protocolo para o cuidado de enfermagem com abscesso de parede pós-cesárea. Dos 15 textos encontrados na BDENF, 8 foram excluídos por estarem duplicados na LILACS. Além destes, quatro dissertações ou teses e um artigo foram excluídos por não estarem disponíveis na íntegra, sendo selecionados somente uma tese e um artigo na BDENF. Na MEDLINE foram localizados dois estudos, porém ambos eram no idioma inglês e estavam indisponíveis na íntegra. $\mathrm{Na}$ SciELO foram encontrados três textos, sendo todos excluídos por estarem repetidos na LILACS. Assim, a amostra final desta revisão integrativa foi composta de 11 estudos, sendo 7 artigos, uma dissertação e uma tese da LILACS ${ }^{(7,9-14,16-17)}$ e dois artigos da BDENF ${ }^{(8,15)}$.

Após a seleção dos estudos, foi elaborado um instrumento para reunir e sintetizar as informações a serem extraídas dos textos. Nele, constavam as seguintes questões: periódico de publicação ou instituição; ano de publicação; referenciais teóricos; abordagem metodológica; características da amostra; objetivos do estudo; principais resultados; e as conclusões e possíveis recomendações para a prática da enfermagem.

A análise dos resultados foi realizada, buscando estabelecer uma interface entre os resultados referentes ao cuidado de enfermagem no AC e a PNAISM ${ }^{(2)}$. Para tanto, foi utilizada a Política associada a outros estudos da área, bem como as ideias dos autores para embasar a discussão.

\section{RESULTADOS}

Primeiramente, apresentam-se as informações extraídas dos textos: periódico de publicação ou instituição; ano de publicação; referenciais teóricos; abordagem metodológica e características da amostra.

A amostra desta revisão integrativa contém nove artigos publicados nos seguintes periódicos: Texto e Contexto Enfermagem ${ }^{(7,10,15)}$, Cogitare Enfermagem ${ }^{(8)}$, Acta Paulista Enfermagem ${ }^{(9)}$, Revista do Instituto de Ciências da Saúde ${ }^{(11)}$, Ciência Cuidado e Saúde ${ }^{(12)}$, Revista da Escola de Enfermagem da USP( ${ }^{(13)}$, Revista da Rede de Enfermagem do Nordeste ${ }^{(14)}$; entre os anos de 2005 e 2010. Além dos artigos, estão inclusas nesta revisão uma dissertação ${ }^{(16)}$, da Universidade do Estado do Rio de Janeiro e uma tese ${ }^{(17)}$, da Escola de Enfermagem de Ribeirão Preto da Universidade de São Paulo publicadas, respectivamente, nos anos de 2009 e 2005.

Com relação ao referencial teórico, quatro estudos explicitaram o referencial adotado, sendo eles: a Teoria do Interacionismo Simbólico ${ }^{(7)}$, a Etnoenfermagem ${ }^{(10)}$, a Teoria do Desenvolvimento Emocional de Winnicott ${ }^{(16)}$ e o referencial de Gênero ${ }^{(17)}$. Com relação à abordagem metodológica, a maioria dos estudos foi qualitati$\mathrm{vo}^{(7-11,13-14,16-17)}$. As puérperas foram o foco predominante de investigação, encontradas em 8 estudos; os demais abordaram a família e os trabalhadores de enfermagem, seja isoladamente ou na mesma pesquisa.

Adiante são abordados os objetivos e os principais resultados, bem como as conclusões e possíveis recomendações para a prática da enfermagem, extraídas dos estudos investigados.

Um estudo ${ }^{(7)}$ objetivou refletir acerca da implementação do modelo de cuidar em enfermagem junto às mulheres e sua família no cotidiano do processo de amamentação, demonstra que é possível leválas a práticas de cuidar hospitalar e domiciliar. Isso evidencia que aplicar um modelo de cuidar é viável, possibilitando promover mudanças nas práticas das mulheres-avós e dos seus familiares frente o processo de amamentação da nutriz.

Outro estudo ${ }^{(15)}$, que objetivou avaliar a satisfação das puérperas com os cuidados de enfermagem recebidos em um AC, indica que todas investigadas relataram satisfação com os cuidados recebidos nesse ambiente. Mulheres com três ou mais gestações e aquelas que vivenciaram o parto vaginal relataram maior nível de satisfação. Os autores alertam para atentar às particularidades individuais das puérperas e repensar o cuidado no aspecto técnico, educacional e humanístico.

Do mesmo modo, outro estudo ${ }^{(8)}$,que objetivou desvelar as percepções sobre a transição ao papel materno entre puérperas primigestas no período pós-parto imediato e delinear estratégias de cuidado de enfermagem transicional que facilitem o alcance ao papel materno, evidencia que a enfermagem deve realizar um cuidado que visualize a mulher como um ser integral e único, respeitando suas necessidades, singularidade, individualidade e limites. Mais do que isso, ela deve ultrapassar o fazer tecnicista e estabelecer uma conduta mais humana e solidária; aspectos esses que devem ser observados desde o processo de formação de forma a contribuir para enfermeiros mais humanos.

Nessa direção, pesquisa ${ }^{(10)}$ cujo objetivo foi compreender a rede de símbolos e significados expressa na vivência do tempo narrativo entre a enfermagem e as famílias, durante o período institucional pós-natal, destaca alguns elementos que podem contribuir para um cuidado mais humano: o uso da ludicidade e a linguagem não-verbal (toque terapêutico, o exercício da paciência, de saber ouvir, de saber o momento de conversar, bem como quando o silêncio é mais bené- 
fico à mulher e aos familiares). Os trabalhadores de enfermagem apontam que é preciso identificar o cliente como um 'outro' envolvido em excepcionalidades. Dessa forma, os autores buscam abrir espaço para a reflexão acerca do fazer da enfermagem, com vistas a ultrapassar o modelo hegemônico estabelecido.

Diferentemente desses resultados, pesquisa ${ }^{(9)}$ que teve como objetivo compreender os significados de ser mãe HIV-positivo para trabalhadoras de enfermagem e para mulheres soropositivas e identificar as similaridades e contrastes presentes no interior desses significados revela atitudes preconceituosas e discriminatórias dos profissionais, pois estes entendem que o papel de mãe é incompatível com o fato dessa ser HIV positivo. Já as puérperas revelam a necessidade de maior compreensão, pois sentem-se felizes e realizadas ao serem mães. Os autores salientam a necessidade de reconhecer essas mães como sujeitos de direitos, como cidadãs, em sua totalidade, individual e social. Isso pode contribuir para minimizar o estigma, preconceito e/ou discriminação social e, deste modo, favorecer a humanização do cuidado à essa clientela.

Com relação ao aleitamento materno, estudo ${ }^{(11)}$ que objetivou identificar e analisar fatores de risco para o desmame precoce na perspectiva das puérperas evidenciou o desconhecimento delas quanto ao benefício da amamentação para a mãe. Além disso, identificou a falta de orientação no pré-natal como um dos fatores que influencia o desmame precoce, dentre outros. Portanto, é necessário que o cuidado de enfermagem incentive $o$ aleitamento materno, por meio do esclarecimento dos benefícios mãe/bebê durante o pré-natal e no puerpério imediato.

Outro estudo ${ }^{(12)}$, cujo objetivo foi levantar os fatores de risco para o "vínculo mãe/bebê" prejudicado, identificados por enfermeiros que atuam em unidade materno-infantil; identificar as ações de enfermagem empregadas para fortalecer o vínculo mãe-bebê e prevenir transtornos que possam prejudicar essa relação, destaca o despreparo dos profissionais $(39,4 \%)$ como fator de risco e ações de enfermagem que podem favorecer esse vínculo foram incentivos ao $\mathrm{AC}(87,9 \%)$ e à amamentação na primeira hora de vida do $\mathrm{RN}(72,7 \%)$.

Um estudo ${ }^{(13)}$ que objetivou identificar as necessidades de puérperas que demandam cuidados de enfermagem e compreender como elas percebem os cuidados, durante sua permanência no $\mathrm{AC}$, evidencia a submissão das mulheres ao poder institucional. Em face disso, elas demonstram necessidade de acolhimento, de cuidados físicos e ambientais que lhes pro- porcionem maior conforto durante sua internação. Os autores ressaltam que a Enfermagem pode contribuir para a formação de uma consciência de gênero e na construção de indicadores qualitativos de avaliação da assistência à mulher no período puerperal.

Outra pesquisa ${ }^{(14)}$, cujo objetivo foi conhecer percepções de puérperas adolescentes sobre a assistência de enfermagem no AC, aponta percepções negativas, tais como a desumanização do cuidado e a ausência de um espaço exclusivo para as puérperas adolescentes. Ressalta-se a importância da conversa e da escuta de forma a atender as necessidades emocionais das puérperas, principalmente, as adolescentes. As diferentes percepções podem indicar a necessidade de adotar o Processo de Enfermagem como forma de individualizar o cuidado.

Em um estudo ${ }^{(16)}$ que objetivou descrever as vivências da mulher no espaço do AC destaca esse ambiente como desfavorável para propiciar o cuidado materno, embora as mães se mostrassem disponíveis para isso. Elas sentem-se abandonadas pelos profissionais de enfermagem, embora a ausência de acompanhantes seja um fator contribuinte para isso. Propõe-se que as instituições públicas repensem o espaço do AC como ambientes facilitadores do cuidado materno, valorizando a mulher e sua família como protagonista do cuidado.

Outra pesquisa ${ }^{(17)}$, cujos objetivos foram identificar a visão das puérperas sobre os cuidados de enfermagem recebidos, analisar as necessidades das puérperas que demandam cuidados, e identificar subsídios de gênero para o cuidado de enfermagem à puérpera durante sua internação no $\mathrm{AC}$, demonstra nos resultados a insatisfação no que tange ao acolhimento, à escuta, à educação em saúde, ao exame físico, ao acesso ao serviço de saúde, à falta de acompanhante, ao ambiente, à alimentação e à auto-imagem. Foram propostos indicadores de gênero com vistas a uma assistência de enfermagem e de saúde com mais qualidade e menos iniquidade.

A seguir, os textos selecionados são analisados quanto aos resultados concernentes ao cuidado de enfermagem no espaço do AC e sua relação com a PNAISM.

\section{DISCUSSÃO}

Nos estudos analisados percebem-se diferentes percepções do cuidado de enfermagem no AC, tanto na ótica das usuárias ${ }^{(7-11,13-17)}$ de saúde quanto da Enfermagem $^{(10,12)}$. Alguns estudos ${ }^{(7-8,10,15)}$ demonstram aspectos positivos do cuidado de enfermagem destacando, inclusive, alguns elementos que podem contribuir para 
qualificar e humanizar a assistência à mulher: incluir no cuidado a rede de relacionamentos da mulher; sistematizar o cuidado de enfermagem; compreender a mulher como um ser integral; utilizar a ludicidade, o toque terapêutico e a linguagem não-verbal; além de atentar para as particularidades de cada ser humano.

Contudo, outros estudos ${ }^{(9,11-14,16-17)}$ demonstram a necessidade de repensar o fazer da Enfermagem, ainda predominantemente tecnicista e fragmentado, no qual a subjetividade, a individualidade e a vontade da mulher são relegadas ao segundo plano ou mesmo ignoradas. Com relação aos aspectos negativos evidenciou-se: o preconceito e a discriminação da Enfermagem às mães HIV positivo; a falta de orientação acerca dos benefícios da amamentação para a nutriz; o despreparo dos profissionais para favorecer o vínculo mãe-bebê; a submissão das puérperas ao poder institucional; o ambiente desfavorável para promover o conforto da puérpera; e a falta de humanização no cuidado de enfermagem; e o sentimento de abandono expressado pelas puérperas, decorrente da presença mínima dos profissionais de enfermagem e da ausência de familiares no $\mathrm{AC}^{(9,11-14,16-17)}$.

Os estudos analisados permitem inferir que as mulheres não são tratadas como sujeitos de direitos, cujas vontades não são respeitadas ou sequer ouvidas, sendo agentes passivos nessa relação de cuidado. Em suas falas, revela-se a necessidade de cuidados que vão além de aspectos relativos ao corpo físico, e estendemse à humanização da assistência e ao ambiente com condições mínimas para oferecer conforto. Assim, surge a necessidade de reorganizar as práticas de saúde em busca do acolhimento e do respeito à mulher como sujeito de direitos conforme preconiza a PNAISM ${ }^{(2)}$.

A PNAISM é norteada pelo princípio da humanização, a qual se caracteriza por "um processo contínuo e demanda reflexão permanente sobre os atos, condutas e comportamentos de cada pessoa envolvida na relação" "(2:60). Sendo assim, exige mais do que prestar um cuidado técnico ao outro, mas repensar o seu fazer, a fim de promover o seu autoconhecimento e, com isso, melhor compreender o outro nas suas diferenças e singularidade, auxiliando no processo saúde-doença "sem procurar impor valores, opiniões ou decisões"(2:60).

Nesse sentido, assim como um dos estudos indicou, a maternidade implica a transição do papel de mulher para o papel de mãe. Para tanto, a mulher precisa adaptarse, tanto física quanto emocionalmente, às alterações próprias do ciclo gravídico-puerperal. Essa assertiva corrobora outros autores que afirmam que à enfermagem incumbe a função de compreender essas adaptações da mulher e realizar uma avaliação com eficiência, com vistas a auxiliá-la na transição mulher-mãe ${ }^{(18)}$.

Com base no PNAISM entende-se que o cuidado de enfermagem à mulher, no $\mathrm{AC}$, requer uma compreensão ampliada acerca do seu contexto de vida, do momento de transição vivenciado, das necessidades expressas, bem como da sua particularidade como um ser único, capaz de realizar suas escolhas, de forma consciente e responsável. Isso implica estabelecer relações singulares, com fortalecimento do acolhimento e humanização do cuidado, o que foi identificado na minoria dos estudos investigados.

Um aspecto concernente ao cuidado no $\mathrm{AC}$ e que merece destaque é a assistência às mães HIV positivo, pois estudo ${ }^{(9)}$ demonstra práticas de enfermagem permeadas por atitudes preconceituosas e discriminatórias. Isso vai ao encontro dos resultados de outro estudo, no qual as mulheres soropositivas para o vírus da Aids relatam sofrer pelo estigma da doença. Por isso, em alguns casos, para se preservarem, elas ocultam a doença ${ }^{(19)}$. Essas práticas contrariam as ações preconizadas pela PNAISM, a qual prima pelo respeito às diferenças da mulher, sua individualidade e integralidade ${ }^{(2)}$.

Diante do exposto, a PNAISM traz, em seu conteúdo, os princípios humanísticos que devem nortear as ações em saúde no cuidado profissional à mulher, seja no contexto do AC ou em qualquer outro ambiente de atuação profissional. Assim, essa parece ser a melhor estratégia para aproximar as ações de saúde às necessidades das gestantes, puérperas, recém-nascidos e seus familiares, o que pode favorecer a melhoria de saúde desse grupo populacional.

\section{CONSIDERAÇÕES FINAIS}

Os resultados dessa revisão integrativa da literatura permitiram discutir acerca do cuidado de enfermagem realizado no ambiente de AC. O cuidado, em sua maioria, foi identificado como insatisfatório, tendo em vista que muitas necessidades da mulher não foram supridas em decorrência do descuidado profissional ou mesmo de um ambiente desfavorável, contrariando a PNAISM.

Ademais, foram assinalados aspectos positivos do cuidado de enfermagem, os quais podem e devem ser valorizados e, aspectos negativos que devem ser repensados e, sobretudo, modificados de forma a atender as reais necessidades de saúde das mulheres, contribuindo para o alcance de um cuidado mais humanizado e qualificado no ciclo gravídico-puerperal.

Por isso, considera-se que a enfermagem e demais profissões da saúde permanecem com o desafio de 
humanizar e qualificar a assistência à mulher, no ciclo gravídico-puerperal, visto que ainda encontram-se profissionais impregnados de preconceitos, atitudes discriminatórias e, sobretudo, desumanas.

\section{REFERÊNCIAS}

1. Ministério da Saúde (BR). Área Técnica de Saúde da Mulher. Parto, aborto e puerpério: assistência humanizada à mulher. Brasília: Ministério da Saúde; 2001.

2. Ministério da Saúde (BR). Secretaria de Atenção à Saúde. Departamento de Ações Programáticas Estratégicas. Política Nacional de Atenção Integral à Saúde da Mulher: princípios e diretrizes. Brasília: Ministério da Saúde; 2009.

3. Ministério da Saúde (BR). Programa de Assistência Integral à Saúde da Mulher: bases de ação programática. Brasília: Ministério da Saúde; 1984.

4. Ministério da Saúde (BR). Área Técnica de Saúde da Mulher. Política Nacional de Atenção Integral à Saúde da Mulher: princípios e diretrizes. Brasília: Ministério da Saúde; 2004.

5. Ministério da Saúde (BR). Secretaria de Atenção à Saúde. Departamento de Ações Programáticas Estratégicas. Manual dos comitês de mortalidade materna. $3^{\text {a }}$ ed. Brasília: Ministério da Saúde, 2007.

6. Cooper HM. The integrative research review: a systematic approach. Beverly Hills (CA): Sage Pulications; 1984.

7. Teixeira MA, Nitscheke RG. Modelo de cuidar em enfermagem junto às mulheres-avós e sua família no cotidiano do processo de amamentação. Texto Contexto Enferm. 2008;17(1):183-91.

8. Alves AM, Gonçalves CSFG, Martins MAM, Silva ST, Auwerter TCA, Zagonel IP. A enfermagem e puérperas primigestas: desvendando o processo de transição ao papel materno. Cogitare enferm. 2007;12(4):416-27.

9. Monticelli M, Santos EKA, Erdmann AL. Ser-mãe HIVpositivo: significados para mulheres HIV positivo e para a enfermagem. Acta paul. enferm. 2007;20(3):291-8.

10. Monticelli M, Elsen I. Quando o tempo narrativo ultrapassa o tempo da clínica: um modo de cuidar em enfermagem no período pós-natal. Texto Contexto Enferm. 2005;14(2):167-76.
11. Silva AV, Oliveira DM, Grei EVE, Gonçalves PC, Gesteira ECR. Fatores de risco para o desmame precoce na perspectiva das puérperas - resultados e discussão. J. Health Sci. Inst. 2009;27(3):220-5.

12. Mendes APD, Galdeano LE. Percepção dos enfermeiros quanto aos fatores de risco para o vínculo mãe-bebê prejudicado. Cienc. cuid. Saúde. 2006;5(3):363-71.

13. Almeida MS, Silva IA. Necessidades de mulheres no puerpério imediato em uma Maternidade pública de Salvador, Bahia, Brasil. Rev. Esc. Enferm. Usp 2008; 42(2): 347-54.

14. Nóbrega LLR, Bezerra FPF. Percepções de puérperas adolescentes frente à assistência de enfermagem no Alojamento Conjunto. Rev. Rene. 2010; 11(Número Especial):42-52.

15. Odinino NG, Guirardello EB. Satisfação da puérpera com os cuidados recebidos no Alojamento Conjunto. Texto Contexto Enferm. 2010;19(4):682-90.

16. Pilotto DTS. As vivências de mulheres no cuidado materno [dissertação]. Rio de Janeiro (RJ): Universidade do Estado do Rio de Janeiro; 2009.

17. Almeida MS. Assistência de enfermagem à mulher no período puerperal: uma análise das necessidades como subsídios para a construção de indicadores de gênero [tese]. Ribeirão Preto (SP): Universidade de São Paulo; 2005.

18. Catafesta F, Zagonel IPS, Martins M, Venturi K. A amamentação na transição puerperal: o desvelamento pelo método de pesquisa cuidado. Esc. Anna Nery. 2009;13(3):609-16.

19. Moura EL, Kimura AF, Praça NS. Ser gestante soropositivo para o vírus da Imunodeficiência Humana: uma leitura à luz do Interacionismo Simbólico. Acta paul. enferm. 2010;23(2):206-11. 